\title{
Impulsividad, funciones ejecutivas y aprendizaje: Una relación para reflexionar
}

\author{
Impulsivity, executive functions, and learning: A relationship to think about \\ Paola Almitra Vázquez-Moreno ${ }^{a}$
}

\begin{abstract}
:
Subclinical impulsivity is defined as a stable personality trait in which individuals who manifest it present risky behaviors and poor decision making. The presence of impulsivity is associated with suboptimal performance in various cognitive processes, such as working memory, cognitive flexibility and inhibition, processes that facilitate the organization and regulation of behavior, and therefore have a negative impact on different areas of the individual's daily life, such as work, social and academic life. The purpose of this paper is to make a narrative review of the concept of impulsivity and executive functioning in relation to learning in undergraduate students.
\end{abstract}

Keywords:

Impulsivity, Executive Function, Self-regulated learning

\section{Resumen:}

La impulsividad subclínica, es definida como un rasgo estable de la personalidad en el cual los individuos que la manifiestan presentan conductas de riesgo y una toma de decisiones deficiente. La presencia de impulsividad se asocia a un desempeño subóptimo en diversos procesos cognitivos, como la memoria de trabajo, la flexibilidad cognitiva y la inhibición, procesos que facilitan la organización y la regulación de la conducta, por lo que impactan de forma negativa en distintos ámbitos de la vida cotidiana del individuo, como el laboral, el social y el académico. El presente trabajo tiene como propósito hacer una revisión narrativa del concepto de impulsividad y el funcionamiento ejecutivo en relación con el aprendizaje en estudiantes de nivel superior.

\section{Palabras Clave:}

Impulsividad, Funciones Ejecutivas, Aprendizaje Auto-regulado

\section{Introducción}

La impulsividad es un constructo que la psicología ha estudiado desde diferentes perspectivas $y$, paradójicamente, está lleno de confusión. El primer problema es el uso coloquial del término. De forma común, es un adjetivo que se usa de forma negativa, en general se refieren como "impulsiva" a cualquier persona que, a juicio del espectador, reacciona de forma precipitada (y equivocada) en respuesta a los estímulos que le rodean. Entonces, impulsivo es aquella persona que aparentemente, es incapaz de resistir la urgencia de actuar: es el niño que toma el dulce a pesar de que se le solicitó no hacerlo; es el adolescente que no entró a clases, a pesar de saber que podría repetir el curso; son los jóvenes que tienen relaciones sexuales sin ningún tipo de precaución, a pesar de no desear un embarazo. Todas estas acciones son categorizadas como impulsivas en el colectivo social y tienen en común que los protagonistas, tenían conocimiento de las posibles consecuencias negativas que acarreaban sus acciones.

Tomando en consideración que el uso coloquial del término ha traspasado hacia la psicología popular, y resulta comun su atribución a múltiples conductas, diferentes entre si, es importante reflexionar sobre el uso del constructo y delimitar sus atribiuciones en la población. Para ello, haremos una revisión general del constructo, para despues revisar los estudios que lo relacionan con déficits en el funcionamiento ejecutivo para finalmente describir las dimensiones del aprendizaje que se podrían ver afectadas entre los estudiantes com impulsividad subclínica.

\footnotetext{
a Autor de Correspondencia, Universidad Autónoma del Estado de Hidalgo, https://orcid.org/0000-0002-0156-4735, Email: paola_vazquez@uaeh.edu.mx
} 


\section{Impulsividad}

La palabra "impulsividad" es un sustantivo abstracto que deriva de la palabra "impulso" y hace relación al movimiento o reacción de surge del interior del individuo. Esta referencia asocia a la impulsividad al movimiento excesivo en los individuos o, a una falta de control en las acciones (Logan, 1997). Aunque una mayoría de los estudios se apegan a la idea original del concepto, y sugieren que la impulsividad está asociada principalmente a fallas en la inhibición motora (Correa, et al., 2010; Fallgatter \& Herrmann, 2001) y por lo tanto un control deficitario de la conducta, otros trabajos se centran en otras características como la incapacidad para retrasar la gratificación (Martin et al., 2010), la dificultad para apreciar el paso del tiempo (Berlin et al., 2004) y las dificultades en la regulación emocional (Cyders \& Coskunpinar, 2011). Estas características heterogéneas sugieren la existencia de distintos tipos de impulsividad, por lo que el constructo no puede ser abordado como unitario.

Adicionalmente, las acciones impulsivas no significan que una persona sea impulsiva, por lo que la impulsividad deviene en un concepto más complejo de lo que aparenta. Primero, en la quinta edición del Manual de Diagnóstico y Estadístico de los Trastornos Mentales, DSM por sus siglas en inglés (American Psychiatric Association, 2014), la impulsividad es un síntoma nuclear en diferentes desórdenes como el TDAH y de los trastornos de personalidad del clúster $\mathrm{B}$, específicamente del trastorno de personalidad antisocial y del limítrofe. Además, la impulsividad se menciona como parte de las características que apoyan en el diagnóstico en distintos trastornos como en el trastorno bipolar, en el juego patológico, y en el trastorno por abuso de alcohol y otras sustancias.

Segundo, en el mismo manual, es mencionado como un rasgo de personalidad, caracterizado por "Dejarse llevar por el fragor del momento...; actuar de forma repentina... sin considerar las consecuencias; dificultad para establecer y seguir los planes; sentido de urgencia y conductas de autolesión en virtud de la angustia emocional" (American Psychiatric Association, 2014, p. 767). Por lo tanto, el concepto parece tener una doble función: síntoma en diversos desórdenes y rasgo de personalidad. En relación con esta segunda función, algunos autores establecen que la impulsividad obedece a factores temperamentales, por lo que se considera como un componente importante en diferentes dimensiones de los distintos modelos de personalidad.

Al respecto, Eysenck presentó inicialmente un modelo con dos rasgos dominantes: Extraversión y Neuroticismo, ubico a la impulsividad inicialmente en la primera. De forma posterior, Eysenck incluyó una tercera dimensión a su modelo a la que denominó Psicoticismo, relacionada a características psicopatológicas de la personalidad. Al agregar esta dimensión, la impulsividad se desplazó hacia la dimensión Psicoticismo, aunque algunas facetas que pueden considerarse parte de la impulsividad como la búsqueda de sensaciones y la propensión al riesgo se mantuvieron en el rasgo de Extraversión (Eysenck \& Eysenck, 1977).

Posteriormente, Costa y McCrae propusieron un modelo de cinco factores, llamado Big Five, compuesto por cinco rasgos (Extraversión, Neuroticismo, Amabilidad, Escrupulosidad, Apertura/Intelectual) y con varias facetas pertenecientes a los rasgos principales (Costa \& McCrae, 1992). En este modelo, la impulsividad se encuentra dentro del Neuroticismo, pero otras facetas que comparten características con la impulsividad como la Búsqueda de excitación y el Control de impulsos se ubican respectivamente en Extraversión y Escrupulosidad por lo que, de forma similar al modelo de Eysenck, encontramos que la impulsividad no es un rasgo unitario y homogéneo de la personalidad, sino que agrupa diferentes características cognitivas, conductuales y afectivas que no se acotan a un solo rasgo.

Estas características cognitivas, conductuales y afectivas, se asocian a diferentes conductas de riesgo y reflejan el funcionamiento ejecutivo de los individuos, además de que influyen en los estilos de aprendizaje, por lo que tienen un efecto deletéreo en el logro de las metas y objetivos de los individuos.

\section{Funciones ejecutivas}

Las Funciones Ejecutivas (FEs) se definen como el conjunto de habilidades que hacen posible la regulación de la conducta orientada a objetivos. Estas habilidades permiten evitar las distracciones, mantener en mente las instrucciones para completar una tarea, planificar y organizar la conducta con orientación al futuro, resistir la ejecución de acciones no requeridas, tolerar la frustración y hacer uso de las experiencias pasadas para la solución de problemas inmediatos (Zelazo, et al., 2016).

Aunque no se encuentra uniformidad respecto a qué habilidades se incluyen como parte del funcionamiento ejecutivo, tres habilidades o procesos son los que aparecen con mayor coincidencia en la literatura: Flexibilidad cognitiva, Memoria de trabajo y Control inhibitorio (Diamond, 2013; Miyake, et al., 2000; Zelazo, et al., 2016).

Otras de las habilidades que se incluyen dentro de las FEs son el control de la interferencia, la planificación y la organización. Se seleccionaron como nucleares a la flexibilidad cognitiva, el control inhibitorio y la memoria de trabajo, ya que de estas habilidades se pueden derivar las restantes. Por ejemplo, el control de interferencia hace referencia a la habilidad de suprimir estímulos competitivos y no requeridos para la ejecución de una 
actividad, habilidad que se incluye en el control inhibitorio (Nigg, 2000).

Además, estas tres habilidades (Memoria de trabajo, Control inhibitorio y Flexibilidad cognitiva) muestran una fuerte correlación entre sí contribuyendo al constructo de Funciones Ejecutivas, pero conservan su independencia como variables latentes (Friedman \& Miyake, 2017; Miyake \& Friedman, 2012). Estas habilidades se asocian al funcionamiento de la corteza prefrontal y se desarrollan de la infancia a la edad adulta. En la tercera década de la vida estas habilidades alcanzan su punto máximo de ejecución.

\section{Memoria de Trabajo}

La memoria de trabajo es definida como la habilidad de mantener y manipular información mientras el individuo realiza una tarea cognitiva (Baddeley, 1992), por lo que es una habilidad básica para el logro de otras habilidades complejas como la planificación.

La integridad funcional de la memoria de trabajo permite al individuo mantener la información requerida para cumplir el objetivo y comparar el estímulo actual con el requerido por las instrucciones o metas. La memoria de trabajo facilita la toma de decisiones y la dirección de la conducta orientada a metas, dos habilidades que parecen estar afectadas en la población con niveles más altos de impulsividad.

Romer y colaboradores (2011) realizaron un estudio prospectivo con adolescentes y encontraron que la memoria de trabajo se correlaciona negativamente con la impulsividad; esta relación no se observó entre los puntajes de búsqueda de sensaciones y memoria de trabajo. De forma similar, Panwar y colaboradores (2014) evaluaron la memoria de trabajo mediante resonancia magnética funcional (RMf) en adolescentes impulsivos y encontraron una correlación entre los puntajes de impulsividad motora y la hiperactividad de regiones cerebrales asociadas a procesos cognitivos como la memoria de trabajo y la inhibición.

Otros trabajos ponen en evidencia el papel de la memoria de trabajo como mediador en el desarrollo de diferentes adicciones, entre ellas el consumo de alcohol (Ellingson, et al., 2014). Además, el entrenamiento en memoria de trabajo ha mostrado mejorar la autorregulación de la conducta en pacientes en ex consumidores de metanfetaminas (Brooks, et al., 2017)

Existen pocos estudios que consideren en su muestra poblaciones con impulsividad subclínica, ya que al ser un síntoma de diversas psicopatologías, las poblaciones estudiadas presentan diversos desórdenes, el más común es el abuso de sustancias lo que hace difícil establecer si las diferencias encontradas en la memoria se encontraban previamente o son consecuencia del consumo de sustancias de abuso.

\section{Control inhibitorio}

El control inhibitorio suprime respuestas predominantes, no requeridas o inapropiadas, así como inhibe estímulos externos e internos que interfieren con la realización de una tarea (Bunge, et al., 2002; Nigg, 2000). Evolutivamente, la maduración del control inhibitorio impacta favorablemente la vida del niño ya que le permite focalizar su atención y planificar sus acciones (Fuster, 2002), habilidad relevante para las interacciones sociales y el éxito en la ejecución de conductas orientadas a metas. En el proceso de inhibición intervienen el sistema motor y un conjunto de estructuras corticales prefrontales y parietales que llevan a cabo distintos procesos cognitivos. Garavan y colaboradores (2006) describieron que en el control inhibitorio motor participan dos redes, la primera es una red fronto-parietal, que involucra áreas parietales para la asignación de la atención al estímulo y su evaluación; y la segunda es la red cíngulo-opercular que incluye al cíngulo anterior, la ínsula, la corteza anterior prefrontal y el tálamo, esta red se encarga de la inhibición de la respuesta, la evaluación afectiva de los estímulos y el establecimiento de la relevancia del estímulo en relación con el objetivo.

El proceso más estudiado en relación con la impulsividad es el control inhibitorio, a partir del supuesto que, en los individuos con mayor impulsividad, los errores surgen a partir de la respuesta rápida y precipitada, en la que no se realiza una evaluación adecuada del contexto y los requerimientos conductuales para lograr el o los objetivos propuestos (Basar, et al., 2010). Aunque la relación entre los déficits en el control inhibitorio y la impulsividad parece evidente al sentido común, en trabajos experimentales la evidencia no es homogénea, y muchos trabajos no encuentran diferencias en el tiempo de reacción entre los participantes con mayor puntaje de impulsividad que en aquellos catalogados como no impulsivos (Lijfijt, et al., 2004; Vázquez-Moreno, et al., 2019) por lo que es probable que los errores no se relacionen de forma directa con la velocidad de respuesta, sino con la falta de evaluación del contexto o la situación.

\section{Flexibilidad cognitiva}

La flexibilidad cognitiva o capacidad de cambio, hace referencia a la habilidad de cambiar entre diferentes sets cognitivos (Miyake \& Friedman, 2012), es decir adaptar las estrategias y la conducta para hacer frente a cambios inesperados en el ambiente. La flexibilidad cognitiva es un concepto más complejo con base en la inhibición y la memoria de trabajo (Diamond, 2013), nos permite analizar las situaciones sobre algo desde una perspectiva diferente, cambiar de estrategia cuando tratamos de resolver un problema y enfrentamos un cambio en el 
contexto, finalmente nos permite aprovechar las oportunidades que nos rodean.

La integridad funcional de la flexibilidad cognitiva depende de la interacción entre múltiples regiones cerebrales, algunas anatómicamente separadas y otras tantas interconectadas de forma local. Entre éstas, la red frontoparietal, en la que se incluyen las cortezas prefrontal ventrolateral y dorsolateral, áreas anteriores del cíngulo, las cortezas parietales superior e inferior, el núcleo caudado y el tálamo (Dajani \& Uddin, 2015), áreas también involucradas en la memoria de trabajo y el control inhibitorio.

La flexibilidad cognitiva tradicionalmente se evalúa mediante diferentes tareas como la prueba de cartas Wisconsin (WCST), tareas que lleven al participante a alternar entre dos tipos de estímulos como nombres de animales y nombres de frutas, consonantes y vocales o números pares o nones.

\section{La influencia de las funciones ejecutivas en el aprendizaje}

Las FEs tienen efectos directos e indirectos en el aprendizaje. En población neurotípica, la influencia directa de las FEs sobre el aprendizaje es evidente durante la infancia y posiblemente se extienda hasta la pubertad, mientras que la influencia indirecta, está relacionada a aspectos motivacionales y emocionales, por lo que podría tener un impacto a lo largo de la vida del individuo.

De forma directa, las FEs permiten que el alumno se mantenga en su lugar, regule la atención, inhiba distractores, recuerde y siga las reglas, y finalmente, adopte nuevas perspectivas (Zelazo, et al., 2016), estas acciones permiten la regulación interna de la conducta, por lo que las FEs generan las condiciones necesarias para que el niño adquiera nuevos conocimientos y conviva de forma adecuada con sus pares y profesores.

La influencia directa de las FEs sobre el logro académico tiene mayor evidencia empírica en la infancia y preadolescencia. Se ha documentado que las FEs son indispensables para la construcción, comprensión y aplicación de conceptos tanto científicos como matemáticos (Vosniadou, et al., 2018). Además, muestran una fuerte asociación con el logro de competencias matemáticas y lingüísticas (Best, et al., 2011).

Otra fuente de evidencia empírica de la influencia de las FEs sobre las habilidades académicas se encuentra en los entrenamientos diseñados con el propósito de mejorar el funcionamiento ejecutivo. Entre la variedad de entrenamientos, existen programas completos dirigidos a entrenar al menos tres de las FEs mencionadas previamente y otros que se concentran en una sola función, ya sea la memoria de trabajo o la inhibición.

De forma general, los entrenamientos enfocados a fortalecer la memoria de trabajo han reportado un efecto de transferencia a otros procesos cognitivos (Thorell, et al., 2009) y a las habilidades académicas (Loosli, et al., 2011) con efectos duraderos en la inteligencia fluida (Peng, et al., 2017).

En lo que respecta a los entrenamientos en inhibición, la evidencia comportamental es escasa. Esto puede deberse a que los programas de entrenamiento se realizan usualmente en niños y la inhibición es una habilidad incipiente en la infancia y que mejora progresivamente hasta llegar a la adultez temprana. El desarrollo prolongado de la inhibición se asocia a la maduración de la corteza prefrontal y el cíngulo anterior, estructuras que constituyen su base anatómica (Bunge, et al., 2002), y considerando que la función muestra mejor desarrollo en la adolescencia y la edad adulta, es posible que los beneficios de los entrenamientos en control inhibitorio solo sean visibles, cuando este se realiza en púberes.

En lo concerniente a la influencia indirecta de la FEs sobre la educación, esta se refiere a aspectos motivacionales. Un buen funcionamiento ejecutivo facilita el proceso de aprendizaje en el niño, esto se vincula con una mayor motivación hacia las actividades escolares, mayor sensación de logro y mejor autoestima en el alumno (Zelazo, et al., 2016). Adicionalmente a los efectos afectivos y motivacionales, existe evidencia empírica que asocia el funcionamiento ejecutivo se asocia empíricamente con la estrategias de aprendizaje autorregulado en estudiantes de distintos niveles educativos (Fuchs, et al., 2003; Garner, 2009), en este sentido las FEs, apoyan a las estrategias de automonitoreo y establecimiento de metas a través de la inhibición y la flexibilidad cognitiva.

Al respecto, el aprendizaje autorregulado comprende al conjunto de procesos que facilitan el desarrollo de las habilidades académicas, por lo que el constructo hace referencia a una variedad de procesos guiados por la metacognición, mediante los cuales los alumnos demuestran control sobre su cognición, sus motivaciones, su conducta y el ambiente (Follmer \& Sperling, 2016). Estas habilidades son fundamentales en los alumnos de educación superior, debido a los requerimientos de autogestión del conocimiento y la necesidad del aprendizaje activo.

A diferencia de los niños y adolescentes, en los adultos jóvenes se asume que estas habilidades regulatorias se encuentran desarrolladas, en consideración de la madurez biológica de la corteza prefrontal, por lo que se espera que de forma consciente adapten su conducta a los requerimientos del medio, organicen sus actividades y autogestionen su conocimiento.

En los alumnos de nivel superior, el funcionamiento ejecutivo es fundamental para la planeación de las actividades, el establecimiento de prioridades y la 
organización de las acciones requeridas para la elaboración de tareas y proyectos dentro de los plazos solicitados y con las características solicitadas. Esto toma relevancia, considerando que los estudiantes de educación superior se enfrentan de forma constante a periodos con plazos para entregas de trabajos de múltiples asignaturas. No obstante, es común observar entre los alumnos universitarios conductas evitativas, procrastinación, así como fallos en las estrategias regulatorias, en la planeación y organización. Estos fallos representan una amenaza a su formación universitaria y podrían estar asociados a diversos factores sociales, económicos e invariablemente, a las diferencias individuales.

\section{Conclusiones}

En conclusión, aunque no existe evidencia que asocie de forma clara los rasgos de impulsividad con déficits en funcionamiento ejecutivo, es claro que se requiere estudiar con mayor profundidad la relación entre estos dos constructos. Por una parte, la falta de evidencia conductual de déficits en funciones ejecutivas específicas (memoria de trabajo, flexibilidad cognitiva e inhibición) entre los individuos más impulsivos, puede estar asociada a problemas metodológicos.

Primero, es común observar que los estudios realizan la clasificación de los participantes por los niveles de impulsividad referida a través de escalas y cuestionarios, por lo que la clasificación no se basa en criterios objetivos, sino en la apreciación del mismo sujeto. Segundo, los estudios se realizan principalmente en muestras con psicopatologías, y en muchos casos no se consideran las posibles comorbilidades. Tomando en cuenta que la impulsividad se toma como criterio diagnóstico en diversas patologías, es importante considerar la realización de perfiles neuropsicológicos específicos que permitan conocer con mayor profundidad el funcionamiento ejecutivo en poblaciones subclínicas.

Finalmente, derivado de las características de los individuos que presentan formas subclínicas de impulsividad, es posible que las fallas en el funcionamiento ejecutivo no se deriven de la presencia de fallos en un proceso cognitivo específico, y estás fallas emerjan de los requerimientos para combinar y ejecutar múltiples procesos de forma simultánea, 0 de la asignación de la relevancia a un estímulo o contexto con base a su importancia.

\section{Referencias}

American Psychiatric Association. (2014). Manual diagnóstico y estadístico de los trastornos mentales DSM-5. Madrid: Médica Panamericana.

Baddeley, A. (1992). Working memory. Science, 255(5044), 556-559.
Basar, K., Sesia, T., Groenewegen, H., Steinbusch, H., Visser-Vandewalle, V., \& Temel, Y. (2010). Nucleo accumbens and impulsivity. Prog Neurobiol., 92(4), 533-57.

Berlin, H., Rolls, E., \& Kischka, U. (2004). Impulsivity, time perception, emotion, and reinforcement sensitivity in patients with orbitofrontal cortex lesions. Brain, 127(5), 1108-26.

Best, J. R., Miller, P. H., \& Naglieri, J. (2011). Relations between executive functions and academic achivement from ages 5-17 in a large representative national sample. Learning and individual differences, 21, $327-336$

Brooks, S., Wiemerslage, L., Burch, K., Maiorana, S., Cocolas, E., Schiöth, H. B., . . . Stein, D. J. (2017). The impact of cognitive training in substance use disorder: the effect of working memory training on impulse control in methamphetamine users. Psychopharmacology (234), 1911-1921.

Bryant, F. B., Yarnold, P. R., \& Grimm, L. G. (1996). Toward a measure model of the Affect Intensity Measure: A three factor structure. Journal of Research in Personality, 30, 223-247.

Bunge, S. A., Dudukovic, N. M., Thomason, M. E., Vaidya, C. J., \& Gabrieli, J. D. (2002). Immature frontal lobe contributions to cognitive control in children. Neuron, 33(2), 301-311.

Correa, A., Triviño, M., Perez-Dueñas, C., Acosta, A., \& Lupiañez, J. (2010). Temporal preparation, response inhibition and impulsivity. Brain and Cognition, 73(3), 222-228.

Costa, P. T., \& McCrae, R. R. (1992). Four ways five factors are basic. Personality and individual differences, 13, 653-65.

Dajani, D. R., \& Uddin, L. Q. (2015). Demystifying cognitive flexibility: Implications for clinical and developmental neuroscience. Trends Neurosci., 38(9), 571-578.

Diamond, A. (2013). Executive functions. Annu. Rev. Psychol. (64), 135168.

Ellingson, J. M., Fleming, K. A., Verges, A., Bartholow, B. D., \& Sher, K. J. (2014). Working memory as a moderator of impulsivity and alcohol involvement: testing the cognitive-motivational theory of alcohol use with prospective and working memory updating data. Addict Behav., 39(11), 1622-1631

Eysenck, S. B., \& Eysenck, H. J. (1977). The place of impulsiveness in a dimensional system of personality description. British Journal of Social and Clinical Psychology, 16(1), 57-68.

Fallgatter, A. J., \& Herrmann, M. J. (2001). Electrophysiological assessment of impulsive behavior in healthy subjects. Neuropsychologia, 39(3), 328-333.

Follmer, D. J., \& Sperling, R. A. (2016). The mediating role of metacognition in the relationship between executive function and selfregulated learning. British Journal of Educational Psychology (DOI:10.1111/bjep.12123), 1-17.

Friedman, N. P., \& Miyake, A. (2017). Unity and diversity of executive functions: Individual differences as a window on cognitive structure. Cortex, 86, 186-204.

Fuchs, L. S., Fuchs, D., Prentice, K., Burch, M., Hamlett, C., Owen, R., \& Schroeter, K. (2003). Enhancing third grade student' mathematical problem solving with self-regulated learning strategies. Journal of Educational Psychology, 95(2), 306-315. 
Fuster, J. M. (2002). Frontal lobe and cognitive development. Journal of Neurocytology, 31(3-5), 373-385.

Garavan, H., Hester, R., Murphy, K., Fassbender, C., \& Kelly, C. (2006). Individual differences in the functional neuroanatomy of inhibitory control. Brain Research, 1105(1), 130-42.

Garner, J. K. (2009). Conceptualizing the relations between executive functions and self-regulated learning. The Journal of Psychology, 143(3), 405-426.

Lijffijt, M., Bekker, E., Quik, E., Bakker, J., Kenemans, J., \& Verbaten, M (2004). Differences between low and high trait impulsivity are not associated with differences in inhibitory motor control. Journal of Attention Disorders, 8(1), 25-32.

Logan, G. D., Schachar, R., \& Tannock, R. (1997). Impulsivity and inhibitory control. Psychological Science, 8(1), 60-64.

Loosli, S. V., Buschkuehl, M., Perrig, W. J., \& Jaeggi, S. (2011). Working memory training improves reading processes in typically developing children. Child Neuropsychology, 1-17.

Miyake, A., \& Friedman, N. P. (2012). The nature and organization of individual differences in executive functions: Four general conclusions. Curr Dir Psychol Sci., 21(1), 8-14.

Miyake, A., Friedman, N. P., Emerson, M. J., Witzki, A. H., Howerter, A., \& Wager, T. D. (2000). The unity and diversity of executive function: A latent variable analysis. Cognitive Psychology (41), 49-100.

Nigg, J. T. (2000). On inhibition/disinhibition in developmental psychopathology: Views from cognitive and personality psychology and a working inhibition taxonomy. Psychological bulletin, 126(2), 220246.

Panwar, K., Rutherford, H., Mencl, W. E., Lacadie, C. M., Potenza, M. N., \& Mayes, L. C. (2014). Differential associations between impulsivity and risk-taking and brain activations underlying working memory in adolescents. Addictive Behaviors, 39(11), 1606-1621.

Peng, J., Mo, L., Huang, P., \& Zhou, Y. (2017). The effects of working memory training on improving fluid intelligence of children during early childhood. Cognitive Development (43), 224-234.

Romer, D., Betancourt, L. M., Brodsky, N. L., Giannneta, J. M., Yang, W., \& Hurt, H. (2011). Does adolescent risk taking imply weak executive function? A prospective study of relations between working memory performance, impulsivity, and risk taking in early adolescence. Developmental Science, 14(5), 1119-1133.

Smith, G., \& Cyders, M. (2016). Integrating affect and impulsivity: The role of positive and negative urgency in substance use risk. Drug and alcohol dependence, 163, S3-S12.

Thorell, L. B., Lindqvist, S., Bergman-Nutley, S., Bihlin, G., \& Klingberg, T. (2009). Training and transfer effects of executive functions in preschool children. Developmental Science, 12(1), 106-113.

Vázquez-Moreno, A., González-Garrido, A., \& Ramos-Loyo, J. (2019). Delayed response improves inhibitory control in low- and highimpulsivity adolescents: effects of emotional contexts. International Journal of Psychological Studies, 11(2), 42-53.

Vosniadou, S., Pnevmatikos, D., \& Makris, N. (2018). The role of executive function in the construction and employment of scientific and mathematical concepts that require conceptual change learning. Revue NeuroEducation Journal, 5(2), 62-72.
Zelazo, P. D., Blair, C. B., \& Willoughby, M. T. (2016). Executive functions: Implications for education (NCER 2017-2000). Institute of Education Sciences, U.S. Department of Education. Washington, D.C.: National Center for Education Research. 